Décadrages Décadrages

cınéma, à travers champs Cinéma, à travers champs

$43 \mid 2020$

Abdellatif Kechiche

\title{
Festen (Cyril Teste, 2017), la fusion de deux arts
}

\section{Laure Cordonier}

\section{OpenEdition}

\section{Journals}

Édition électronique

URL : https://journals.openedition.org/decadrages/1602

DOI : 10.4000/decadrages. 1602

ISSN : 2297-5977

\section{Éditeur}

Association Décadrages

Édition imprimée

Date de publication : 1 juillet 2020

Pagination : 168-173

ISSN : 2235-7823

\section{Référence électronique}

Laure Cordonier, «Festen (Cyril Teste, 2017), la fusion de deux arts », Décadrages [En ligne], 43| 2020, mis en ligne le 15 octobre 2021, consulté le 04 avril 2022. URL : http://journals.openedition.org/ decadrages/1602 ; DOI : https://doi.org/10.4000/decadrages.1602 


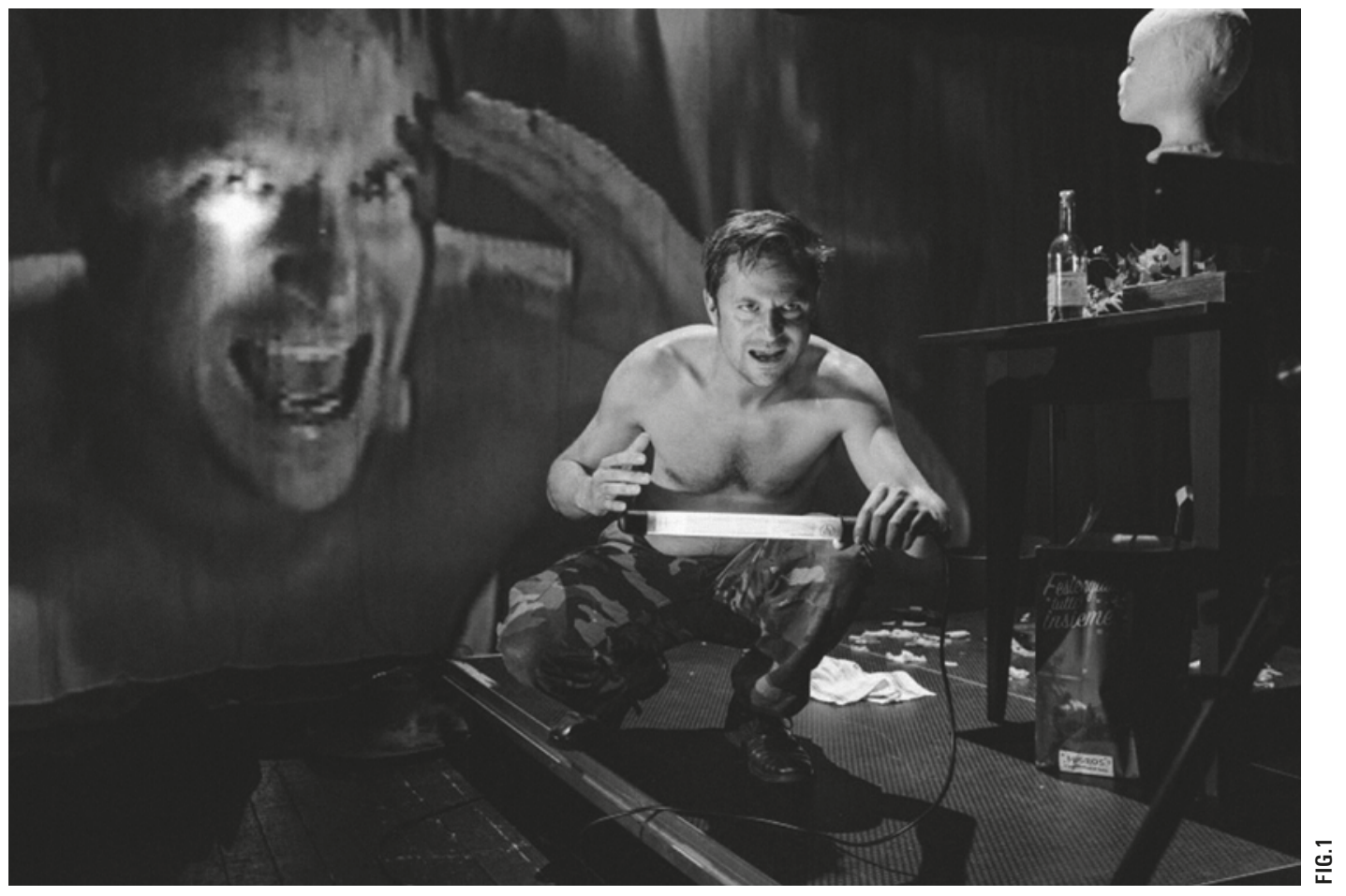


$1 \quad$ Les /doles refaisait vivre sur scène des artistes français décédés du SIDA dans les années 1980-1990.

2 Loin d'être une pièce de théâtre au sens classique du terme, Le Livre d'image consistait en un montage extrêmement succinct de plans de "classiques" du cinéma juxtaposés à des images de différentes guerres ou catastrophes qui ont marqué le $x x^{e}$ siècle. Le film était diffusé sur un écran de télévision de taille moyenne, dans un salon de type familial. Présenter de la sorte un film, non pas dans une salle de cinéma, mais sur un écran de télévision et au milieu d'un décor simulant un salon familial était un acte lourd de sens de la part de Godard, qui, sans doute, illustrait par ce biais sa déception à l'encontre du $7^{\mathrm{e}}$ art, mais aussi critiquait par là le racolage incessant des médias pour capter l'attention du public. début de carrière artistique au théâtre, alors que l'homme est désormais davantage (re)connu pour ses films. En automne 2019, avec Le Livre d'image, Jean-Luc Godard a lui aussi investi Vidy, mais d'une manière bien plus surprenante (et davantage "liée" à l'image animée), puisqu'il a posé une banale télévision au sein d'un décor théâtral de la salle René Gonzalez² ${ }^{2}$ bouleversant ainsi les codes et les frontières entre le visionnement cinématographique, télévisuel et théâtral. À Vidy, le septième art a également été le thème d'une création en 2015, par le biais de Cinéma Apollo de Matthias Langhoff, une œuvre en deux temps/deux médias, composée d'un film de 40 minutes, suivi d'une pièce de près de deux heures dont l'action se passait dans une salle de cinéma.

Mais à Vidy comme ailleurs, la perméabilité entre le théâtre et le cinéma est aussi et souvent d'ordre technique, la présence du dispositif cinématographique sur la scène théâtrale relevant désormais presque de I'habitude. Un exemple parmi tant d'autres: le metteur en scène allemand Nicolas Stemann présentait en 2015 une version atypique du Werther de Goethe, dont les souffrances étaient filmées par le comédien Philipp Hochmair ${ }^{3}$ lui-même et projetées, parfois avec un court décalage temporel, sur un écran placé dans le fond de la scène ${ }^{(\mathrm{Fig} .1)}$. Cette présence d'une caméra sur scène - qui isole des éléments du plateau pour les retransmettre en direct ou en léger différé sur un écran situé, dans la plupart des cas, sur les côtés ou au-dessus de l'espace de la représentation - est presque une constante dans les pièces contemporaines, quitte à lasser parfois les spectateurs ou susciter un effet de saturation.

Si la pièce Festen de Cyril Teste suit cette mode, I'omniprésence des caméras et leur usage dépassent par leur systématique et leur
Werther! (Nicolas Stemann, 2015). 
4 Pour rappel, le récit de Festen se déroule dans un manoir danois où une famille aisée se réunit à l'occasion des soixante ans du père de famille, Helge. Cette soirée sera marquée par le discours de Christian, I'un des fils de Helge, accusant son père de les avoir violés, lui et sa sœur jumelle, durant leur enfance.

5 Sans pour autant renier Dogma dans ses entretiens, Vinterberg s'est rapidement orienté vers un travail nettement plus classique et ne respectant aucunement les règles édictées par le manifeste du mouvement. Ces dernières années, le réalisateur danois a même dirigé des productions à gros budget (Kursk, B/Lux/F, 2018) et effectué un détour par Hollywood (Far from the Madding Crowd, R.-U./E.-U., 2015).

6 Dossier de presse de Festen.

7 Les deux cameramen ne sont donc pas intégrés diégétiquement à la performance, mais se situent dans un ailleurs, aux frontières de la représentation scénique et du public de la salle.

8 Frédérique Roussel, Libération, cité dans le dossier de presse de Festen. subtilité les exemples cités jusqu'ici. Festen est tiré de l'adaptation théâtrale de Bo Hr. Hansen, elle-même élaborée à partir du film homonyme réalisé en $1998^{4}$ par Thomas Vinterberg, réalisateur désormais mainstream ${ }^{5}$ mais qui restera connu dans I'histoire du cinéma pour avoir initié le célèbre mouvement danois Dogma en compagnie de Lars Von Trier. Cette version de Festen est la dernière création du Collectif MxM, qui, sous l'appellation de "performance filmique", développe depuis 2011 des œuvres proposant "une écriture théâtrale qui s'appuie sur un dispositif cinématographique en temps réel et à vue, en décor ou au plateau $"{ }^{6}$. À la manière du manifeste de Dogma, ce collectif français a également édicté sa propre charte de travail. Ses règles stipulent notamment que la performance filmique (dont la source est obligatoirement issue du théâtre) doit être tournée, montée et réalisée en temps réel sous les yeux du public. Il en va de même pour la musique et le son. La performance peut cependant utiliser des images préenregistrées, mais celles-ci ne doivent pas excéder une durée totale de cinq minutes.

L'une des caractéristiques majeures du dispositif cinématographique de Festen réside dans l'omniprésence scénique de deux caméras à l'épaule durant l'ensemble de la représentation. Ces caméras sont tenues par des cameramen visibles sur scène (et qui filment en alternance), et non par les comédiens eux-mêmes ${ }^{7}$. Sur le plateau, la caméra se glisse discrètement parmi les nombreux personnages, les isolant par des plans rapprochés permettant d'insister sur les expressions et la gestuelle. D'un point de vue technique, cette performance exige une précision d'orfèvre et une synchronisation parfaite. Les comédiens et les cameramen doivent en effet adapter leur jeu et leurs mouvements à une coordination optimale pour ne pas troubler le déroulement de I'action. À cela s'ajoute évidemment un lourd et rigoureux travail de régie. L'entreprise est maîtrisée de bout en bout, le film ne s'interrompt jamais, formant ce qui prend l'apparence d'un long plan-séquence d'une heure et demie. Dans les pages de Libération, Frédérique Roussel constate que la pièce se situe «loin de l'effet de mode" consistant à introduire le dispositif cinématographique dans l'espace scénique, dans le sens qu'elle n'est en rien gratuite, comparativement à d'autres pièces ${ }^{8}$. Ce dispositif complexe induit en effet une nouvelle forme de visionnement pour le public, puisque le spectateur se situe dans un espace de représentation hybride; il est libre de regarder soit l'écran de cinéma (fixé en arrière-plan de la scène, au centre, et en hauteur), soit la scène théâtrale, mais cette liberté ne constitue pas nécessairement une ouverture ou un moyen de facilité, elle peut se muer en une hésitation permanente. De ce fait, I'activité ordinaire du spectateur est intense, complexe et même questionnée par cette focalisation plurielle. Du point de vue anthropologique et sociologique, le double dispositif présuppose une faculté attentionnelle multiple de la part des spectateurs disposés à composer avec plusieurs sources d'information. Cette capacité est particulièrement mise en œuvre dans le cas où, de fait, malgré les différences de médiums et de points de vue, c'est forcément une seule et même 
intrigue qui est jouée. Il est en revanche évident que, plus encore que dans une représentation théâtrale standard, le spectateur devient son propre régisseur. Selon les cas, le montage à opérer peut contribuer au plaisir esthétique, dans d'autres il peut fatiguer ou désemparer.

Le sentiment de maîtrise ou le possible désarroi du spectateur résultent probablement de la prise de conscience que, tel qu'il est proposé, le dispositif double ne dilue en aucun cas ni la gravité du propos de Festen ni la participation affective, le pathos, du spectateur mais les intensifie, car le filmé, qui se concentre surtout sur le personnage de Christian (et sur son mal-être), insiste sur les actions et les attitudes qui convergent vers la bouleversante scène du discours d'accusation contre son père. Dans les années 1920, le cinéaste-théoricien Jean Epstein considérait le gros plan comme l'âme du cinéma, en raison de sa capacité à pénétrer l'intériorité des personnages ${ }^{9}$. Force est de constater que cette conception s'actualise puissamment dans la salle de théâtre, par les gros plans serrés sur le visage des personnages principaux de Festen. Ce faisant, la performance filmique sur scène s'inscrit assez fidèlement dans le sillage du film de Vinterberg, qui était lui aussi filmé à l'épaule, dans un style proche de celui des films de famille. En effet, les images de la fête (et du film dans son intégralité) parvenaient au spectateur par le biais d'un enregistrement effectué par une caméra digitale grand public (mini-DV), comme s'il s'agissait d'un film souvenir de l'événement. Cependant, la caméra n'avait aucun fondement diégétique clairement déterminé. En ce sens, la pièce récupère une idée de mise en scène de Vinterberg. Mais, surtout, elle la "prolonge ", grâce à la division de l'espace de représentation offert par le théâtre. En effet, et c'est un autre exemple du constant et double choix que doit opérer le spectateur, la représentation scénique de la performance filmique donne à voir les marges, les à-côtés de l'action dramatique centrale, et par là même, offre une forme d'échappatoire aux spectateurs désireux de suspendre l'intense charge émotionnelle de la pièce.

Le décor et l'éclairage très étudiés de la scène Festen de Cyril Teste présentent d'abord au centre du plateau scénique une imposante salle à manger. Puis, des parois mobiles révèlent alternativement une salle de bains et une chambre à coucher. Enfin, une grande cuisine se découvre côté cour. Par les fenêtres ouvertes, le public accède ainsi à ce qui se passe en cuisine où Olivier Théron, un grand chef français, apprête en direct le menu que dégusteront les personnages ${ }^{10}$. Le réalisme du décor est renforcé par des sensations olfactives, puisque le collectif a collaboré avec Francis Kurkdjian, créateur de parfums à renommée internationale et familier de telles performances. Plus précisément, trois senteurs sont vaporisées dans la salle au fil du spectacle $^{11}$. Avec d'autres manifestations artistiques actuelles qui profitent toutes de l'ère multimédiatique, cette mise en scène, qui tente de stimuler simultanément la vue, l'ouïe et l'odorat des spectateurs, rappelle les réflexions et les mises en scène de type synesthésique qui $\mathrm{s}^{\prime}$ étaient multipliées à la fin du XIX $\mathrm{X}^{\mathrm{e}}$ siècle. Expérimentales, ludiques ou plus ambitieuses, du symbolisme français à l'opéra wagnérien, ces Paris, La Sirène, 1921.

10 Les différents personnages de la pièce sont par ailleurs rapidement rejoints sur scène par deux couples du public. Notons que cette participation du public est anticipée par la production grâce à l'envoi de courriels quelques jours avant les représentations aux Amis du théâtre de Vidy pour proposer à des personnes âgées entre 40 et 50 ans de venir partager le repas des comédiens sur scène.

11 Ces fragrances s'intitulent: "Forêt d'automne quand le soleil se couche", "Fumée extérieure de cheminée» et "Odeur de café et de croissants". 


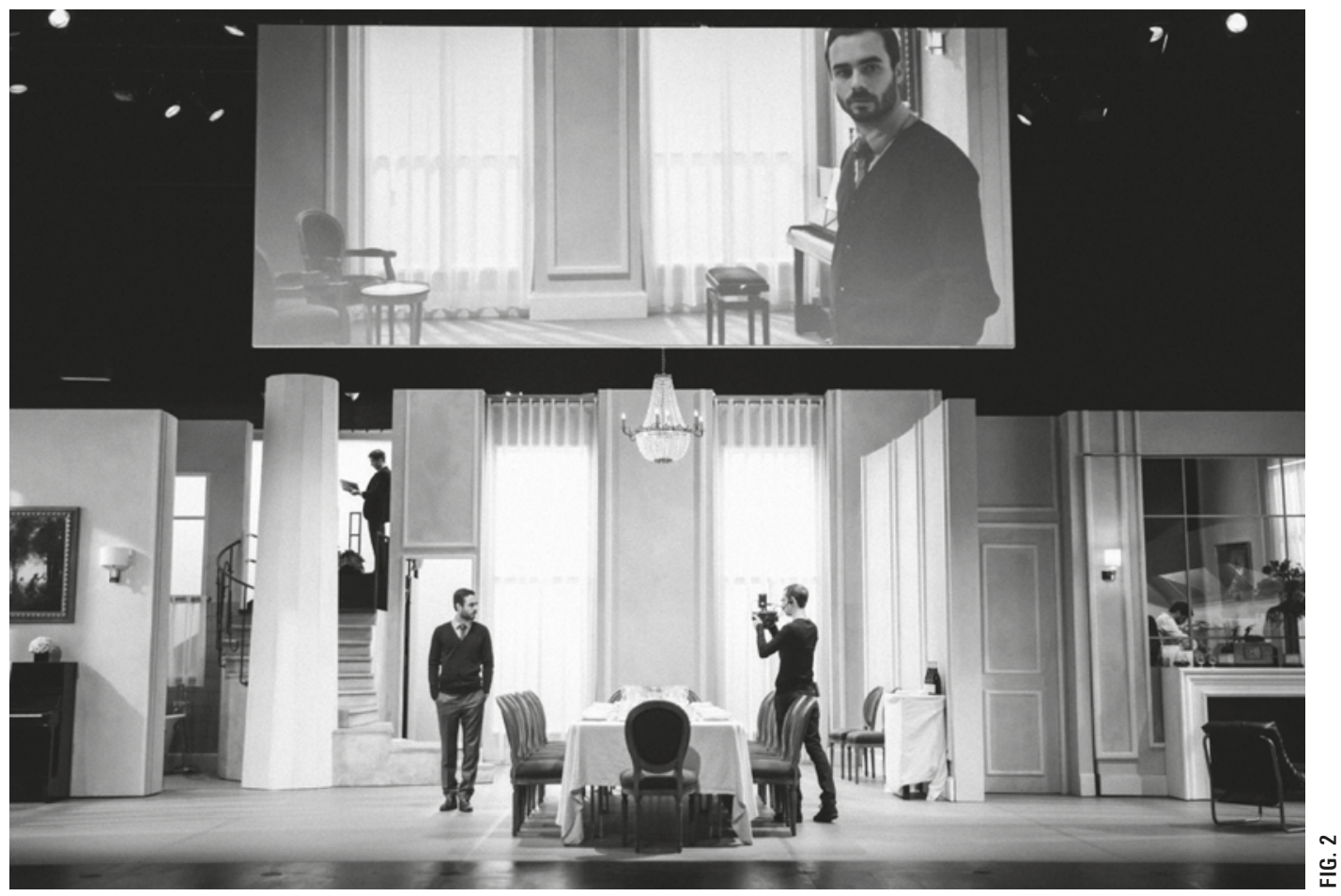


œuvres sollicitant plusieurs sens donneraient accès à des connaissances inédites, sidérantes. N'ayant pas de telles ambitions quand il stimule simultanément plusieurs sens du spectateur - et nous n'en trouverons guère davantage dans l'effet d'immersion des jeux vidéo ou des performances artistiques contemporaines -, le spectacle proposé donne une impression de classicisme, d'économie de moyens, alors que, en réalité, il est d'une rare complexité technique, comme on le vérifie notamment dans le dernier tiers de la performance, lorsque le filmique fusionne avec la mise en scène: seul dans la salle à manger, Christian se souvient avec émotion de sa sœur jumelle Linda, qui elle aussi avait été abusée par leur père et qui s'est donnée la mort un an auparavant. L'évocation crée une rupture dans le plan-séquence filmé en direct. En effet, pour représenter la scène à l'écran, I'un des cameramen filme d'abord en gros plan le visage triste et songeur de Christian, qui repense à la mort brutale de sa sœur jumelle. La caméra s'éloigne ensuite du personnage et cadre le décor de la salle à manger au moyen d'un long travelling latéral. Le filmé coïncide parfaitement avec la scène: le décor est totalement similaire sur l'écran et sur le plateau. Pourtant, par un trucage technique imperceptible pour le spectateur, le film dévoile une femme (la sœur) assise au piano, alors que le personnage, décédé, est absent de la scène. C'est à ce moment et de cette manière que le collectif a employé les cinq minutes préenregistrées qu'autorise sa charte. Cet habile glissement des images retransmises en direct aux plans enregistrés restitue ce que "voit" le frère blessé et révolté. La fusion du dispositif cinématographique et de la représentation théâtrale atteint son acmé. Au-delà de la prouesse technique, le cinéma introduit une image mentale dans le présent, le souvenir que se remémore un personnage.

Une célèbre réflexion de Roland Barthes définit le théâtre comme une machine cybernétique capable de dispenser une multitude d'informations simultanées provenant de sources différentes (décor, costumes, éclairage, jeu des acteurs, etc.). Selon le sémiologue, la pluralité de messages forme une "polyphonie informationnelle ${ }^{12}$. Par leurs travaux mêlant le théâtre au dispositif cinématographique, le collectif MxM mené par Cyril Teste augmente et diversifie le flux des informations que reçoit le spectateur et qu'il doit traiter du mieux qu'il peut, tout en sachant qu'il en néglige sans doute autant qu'il en retient.

Dans I'histoire du cinéma, Festen est considéré comme l'un des films les plus caractéristiques du mouvement Dogma qui cherchait à imposer une nouvelle vision de la production cinématographique et de ses modes d'écriture. Dans I'histoire de la mise en scène, le Festen du collectif $\mathrm{MxM}$ où la tension dramatique est intensifiée - et non disséminée - par une technologie complexe mais discrète, servira sans doute de référence aux mises en scène qui souhaitent faire participer le spectateur au plus haut degré tout en ménageant sa capacité actuelle à composer simultanément avec plusieurs codes et plusieurs médias. signification, Essais critiques, Paris, Seuil, coll. Points, 1981 [1963], p. 258. 